\title{
Analog modeling of sand slope stability with different precipitation conditions
}

\author{
Tangjin $\mathrm{Ye}^{1,2} \cdot$ Qiang Xie $^{1} \cdot$ Ying Wang ${ }^{1,2} \cdot$ Yang $\mathrm{An}^{2} \cdot J_{\text {Jiang Jin }}{ }^{1}$
}

Received: 3 March 2017/Revised: 23 April 2018/Accepted: 14 May 2018/Published online: 5 June 2018

(C) The Author(s) 2018

\begin{abstract}
Water-sand flow triggered by rainfall is the dominant mechanism for instability and failure of sand slopes. To further analyze the stability state of sand on a slope under different rainfall conditions, the initiation conditions and flow characteristics of water-sand flows are studied. Based on the theory of equilibrium forces and hydrological dynamics, a 1:100-scale analog model is built and verified with field observation data. The results indicate three dynamic stabilization stages of the sand slope under different weather conditions: dry sand, wet sand, and water-sand flow. Water-sand flows are triggered easily under short duration and heavy rainfall conditions. The rainfall threshold required to initiate water-sand flow is $4.14 \mathrm{~mm} / \mathrm{h}$. Rainfall amount and duration required to initiate water-sand flow decrease with fine sand content increasing. A sand head that develops at the front of the water-sand flow results in a flow along the edge of the sand debris flow and a "tree root" flow morphology. Modeling results are consistent with theoretical analysis and field observations.
\end{abstract}

Keywords Precipitation - Analog modeling - Sand slope · Water-sand flow $\cdot$ Stability

Tangjin Ye

237492042@qq.com

1 Faculty of Geosciences and Environmental Engineering, Room 1301, Civil Building, Southwest Jiaotong University, No. 111, North 1st Section of Second Ring Road, Chengdu 610031, China

2 College of Engineering, Tibet University, Lhasa 850000, China

\section{Introduction}

The Ranwu-Songzong section of highway G318 in Tibet, China, has numerous active geologic hazards. With an average annual rainfall of 600-700 $\mathrm{mm}$ [1], water-sand flows are very easily triggered by short-term heavy rainfall, which can affect the stability of sand slopes. Based on field investigations conducted in 2015 and statistical analyses of 51 sand slopes, water-sand flow traces were clearly identified in $96.08 \%$ of sand debris flows in the area. As a result, safety management of the Ranwu-Songzong section has been seriously affected (Fig. 1a). The economic loss and maintenance costs associated with the sliding of sand slopes in this section can be up to $10^{6}-10^{7}$ Yuan per year [2]. As field studies are often influenced by heavy rainfall and harsh environments, long-term sustainable observations are difficult. Therefore, our current understanding of sand stability, sand debris flow formation mechanisms due to rainfall, and hazard treatment measures is insufficient (Fig. 1b).

Sand debris flows are mainly concentrated in the European Alps, the Bomi-Ranwu section of the G318 highway in Tibet, and the Tianshan section of the G217 highway in Xinjiang, China. The earliest study on sand debris flows was conducted in 1938, when Sharpe [3] proposed the concept and classification of debris flows. Later, Caine [4] proposed that earthquakes and rainfall were the main factors causing destabilization of sand slopes; Brunsden and Chandler [5] classified landslides into dry sand and wet sand flows according to a moisture content limit of $5 \%$; Gabriels [6] analyzed the influence of slope width on the increase in sand loss; and Yoo [7] studied the use of geotechnical grilles in reinforcing sand-gravel slopes. Furthermore, Pietronero et al. [8] and Mohanty and Dhar [9] studied the self-organized criticality of sand dunes. 


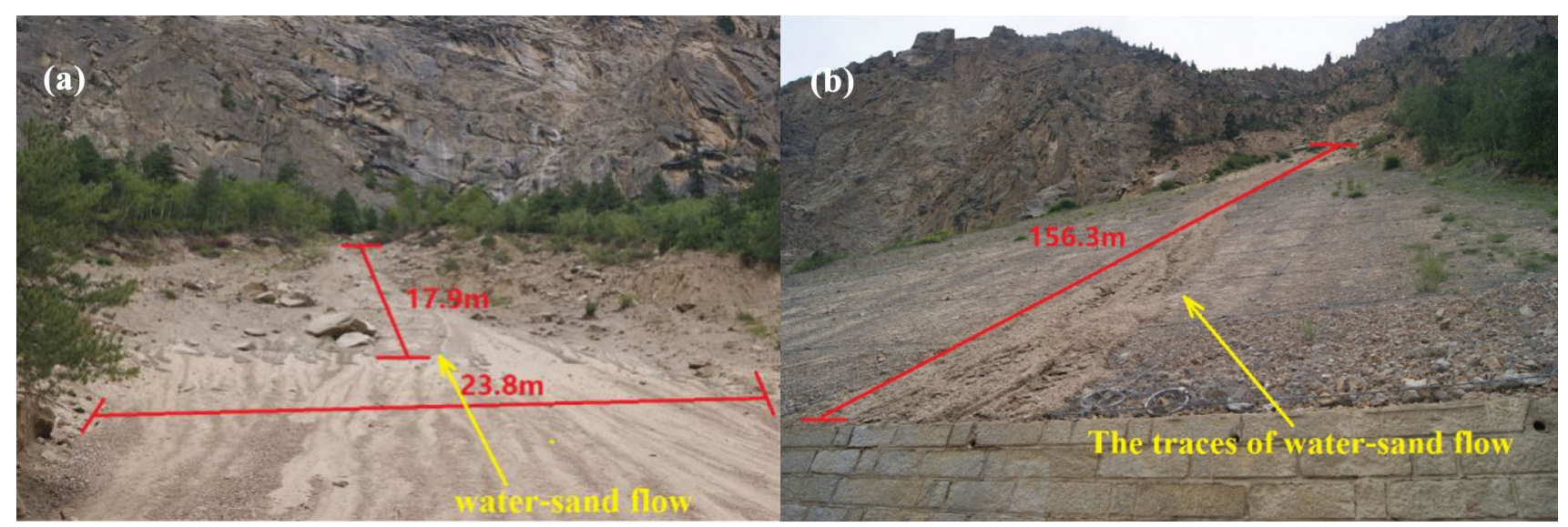

Fig. 1 Water-sand flow initiating (a) and traces of water-sand flow on a sand debris flow after treatment (b)

Research on landslides in China began with Luo et al. [10] who studied debris flows of the G318 highway in Tibet and first proposed the concept of sand slopes. After 2000, many studies were performed on the Zongba section of the G318 highway in Tibet and the Tianshan section of the G217 highway in Xinjiang, mainly focused on the formation mechanisms of dry sand slopes, accumulation states $[11,12]$, flow modes [13, 14], modeling [15, 16], theoretical analysis, and control measures [2, 17]. Wang et al. [12] proposed that gravity and surface water are the main factors inducing sand sliding. Due to the impact of the adverse environment and the limited observation period, however, they did not study water-sand flows formed by surface water, which can affect the stability of sand slopes. Therefore, in this study, using the theory of equilibrium forces and hydrological dynamics, we analyzed and verified the dynamic stabilization processes of sand slopes under different precipitation conditions by combining theoretical analysis with a water-sand flow model. Besides, sand-sliding formation mechanisms and flow characteristics are also studied.

\section{Theoretical analysis on sand debris flow stability}

The main component of sand slopes is sand, of which 94.2-98.2\% is medium and coarse sand with small amounts of gravel. Geotechnical analysis shows that the cohesive force and internal friction angle are affected by the water content of sand (Table 1). Thus, the stability of sand on the slope changes with different meteorological and rainfall conditions.

\subsection{Stability of dry sand and wet sand slopes}

In addition to gravity, the stability of sand is also affected by vibrations and other external forces. The coefficient of slide resistance $k$ of sand on the slope was obtained according to the shear strength between the sand grains and the shear stress on the slope surface, using the following equation [13]:

$k=\frac{G \cos \alpha \tan \varphi+c d}{G \sin \alpha+p_{\mathrm{w}}}$,

where $P_{\mathrm{w}}$ is external force, $d$ is sand diameter, $G$ is gravity, and $\alpha$ is dip of the sand slope.

When it is sunny or cloudy and there is no external force on the dry sand, the force produced by its cohesion $(c d)$ and the external force of sand $\left(P_{\mathrm{w}}\right)$ equals 0 , considering the effect of sand diameter. The repose angle equals the internal friction angle (about $34^{\circ}$ ), and the stability of sand is obtained by Eq. (1): $k=\cot \alpha \cdot \tan \varphi \approx 1$. Thus, the sand debris flow is in an extreme stability condition. When dry sand is influenced by external forces such as vibrations, $P_{\mathrm{w}}>0$ and $k<1$. Sand stability and downward movement are affected by external forces.

When the rainfall amount is less than the threshold value for initiation of a water-sand flow, fast shear parameters in the natural state (Table 1) can be used for samples 4 and 5 , which were collected during light rain. Here, $c=6-8 \mathrm{kPa}$, $\varphi=36^{\circ}-37^{\circ}$, and the thrust of surface water approaches 0 . A value of $k>1$ can be easily obtained, and the sand debris flow is stable. The forces are shown in Fig. 2a. As a result, the stability of sand on the sand debris flow was divided into three stages: dry sand, wet sand, and watersand flow. When rainfall exceeds the initiation threshold value, water-sand flow occurs on the surface of the sand debris flow. The hydrological dynamic features of watersand flows are analyzed in the next section. 
Table 1 Shear strength parameters in a sand debris flow

\begin{tabular}{|c|c|c|c|c|c|c|c|c|c|c|}
\hline \multirow[t]{2}{*}{ Sample no. } & \multirow[t]{2}{*}{ Name } & \multicolumn{3}{|c|}{ Fast shear in natural state } & \multicolumn{3}{|c|}{ Fast shear in drying state } & \multicolumn{3}{|c|}{ Fats shear in saturated state } \\
\hline & & $w(\%)$ & $\varphi\left(^{\circ}\right)$ & $c(\mathrm{kPa})$ & $w(\%)$ & $\varphi\left({ }^{\circ}\right)$ & $c(\mathrm{kPa})$ & $w(\%)$ & $\varphi\left(^{\circ}\right)$ & $c(\mathrm{kPa})$ \\
\hline 1 & Grit & 0.9 & 34.1 & 4 & 0 & 36.4 & 0 & 21.8 & 31.5 & 4 \\
\hline 2 & Medium sand & 1.1 & 34.4 & 6 & 0 & 36.9 & 1 & 22.5 & 30.7 & 7 \\
\hline 3 & Gravel & 0.7 & 34.7 & 5 & 0 & 36.5 & 0 & 9.1 & 33.4 & 1 \\
\hline 4 & Grit & 4.1 & 36.6 & 11 & 0 & 36.3 & 0 & 19.8 & 31.3 & 5 \\
\hline 5 & Medium sand & 4.6 & 37.3 & 12 & 0 & 36.7 & 2 & 21.6 & 30.8 & 6 \\
\hline
\end{tabular}

$w$ is water content, $c$ is cohesion, and $\varphi$ is internal friction angle of sand samples. Weather conditions during collection of samples 4 and 5 were light rain, but sunny for other samples

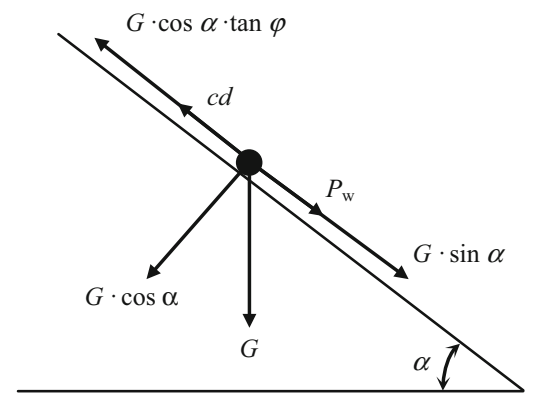

(a)

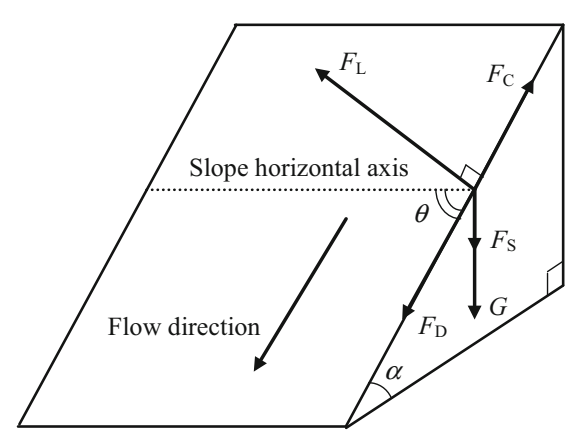

(b)

Fig. 2 Free body diagram of dry sand and green sand (a) and sand in a water-sand flow (b)

\subsection{Hydrological dynamic features of water-sand flows}

When rainfall exceeds the threshold value for initiation of a water-sand flow, the source region starts to collect water and surface runoff occurs on the sand debris flow. The water-sand flow is composed of the surface runoff, the sand in the circulating region, and the front of the accumulation area. In this study, the theory of equilibrium forces and hydrological dynamics [18, 19] of sand was used to analyze the force state and flow characteristics of water-sand flows. Assuming the sand is approximately spherical, the saturated unit weight of sand is $\gamma_{\mathrm{s}}$, and the unit weight of water is $\gamma_{w}$, then the effective gravity on the sand is given by Eq. (2):

$G=\frac{1}{6} \pi\left(\gamma_{\mathrm{s}}-\gamma_{\mathrm{w}}\right) d^{3}$

We assume that the coefficient $\xi$ is related to sand properties such as structure and destiny. The cohesion force of running water on the sand surface is then shown as in Eq. (3):

$F_{\mathrm{C}}=\xi d$.

If the drag coefficient is $C_{\mathrm{D}}$, the lift force is $C_{\mathrm{L}}$, the projection area of the sand in the direction of water flow is $A$, the density of water is $\rho$, and the water flow speed on the sand surface is $v_{0}$, then the impact force and uplift force of sand in the water flow are expressed as in Eqs. (4) and (5), respectively.

$F_{\mathrm{D}}=\frac{1}{2} C_{\mathrm{D}} A \rho v_{0}^{2}$,

$F_{\mathrm{L}}=\frac{1}{2} C_{\mathrm{L}} A \rho v_{0}^{2}$

The seepage pressure $F_{\mathrm{s}}$ is given by Eq. (6):

$F_{\mathrm{s}}=\frac{1}{6} \gamma_{\mathrm{w}} J \pi d^{3}$

where $J$ is the hydraulic gradient of water penetration in the soil.

The forces on the sand in a water-sand flow are shown in Fig. 2b. Because coarse and medium sand from weathered granite dominates the sand composition, the sand is almost inviscid and uniform. The impact force is expressed by the shear component force under critical conditions on a unit area of water weight in the direction of water flow. The impact force of sand on a plane, $\tau$, is obtained by considering the laminar viscous shear and turbulent fluctuation shear of water flow, as follows:

$\tau=\frac{4}{3} \frac{f\left(\gamma_{\mathrm{s}}-\gamma_{\mathrm{w}}+\gamma_{\mathrm{w}} J_{\mathrm{S}}\right) d}{\left(C_{\mathrm{D}}+f C_{\mathrm{L}}\right)\left[f_{1}\left(\frac{U^{*} d}{v}\right)\right]^{2}}$, 
where $J_{\mathrm{S}}$ is the hydraulic gradient, $f$ is the coefficient of friction between the sand grains, $U^{*}=\sqrt{g h J}$ is the shear velocity, and $h$ is the depth of water; $f_{1}\left(\frac{U^{*} d}{v}\right)$ is a function of the Reynolds number, and $v$ is the water flow speed.

When the sand shape approximates sphere, $C_{\mathrm{D}}$ and $C_{\mathrm{L}}$ are associated with the $f_{1}\left(\frac{U^{*} d}{v}\right)$, and the $f$ is constant. Equation (8) can then be obtained using Eq. (7).

$\tau=f_{1}\left(\frac{U^{*} d}{v}\right)\left[\left(\gamma_{\mathrm{s}}-\gamma_{\mathrm{w}}+\gamma_{\mathrm{w}} J_{\mathrm{S}}\right) d\right]$.

When sand is on a slope surface and the slope angle is $\alpha$, the component force of gravity in the slope direction $G \cdot \sin \alpha$ accelerates erosion initiation. The resultant force parallel to the slope is shown in Eq. (9) and the vertical force is shown in Eq. (10).

$F_{1}=\sqrt{\left(F_{\mathrm{D}} \sin \theta+\left(G+F_{\mathrm{S}}\right) \sin \alpha\right)^{2}+F_{\mathrm{D}}^{2} \cos ^{2} \theta}$,

$F_{2}=\left(G+F_{\mathrm{S}}\right) \cos \alpha-F_{\mathrm{L}}$.

From Eqs. (3), (9), and (10), the critical conditions for water-sand flow initiation on a slope can then be obtained as follows:

$\tan \varphi=\frac{F_{1}-F_{\mathrm{C}}}{F_{2}}$.

When the angle between the direction of water flow on a slope and the horizontal axis of the slope is $\theta$ (Fig. 2b), we denote the impact force on the slope by $\tau^{\prime}$. The seepage pressure and cohesion of sand are relatively small and can be neglected because the sand is on a slope. One can obtain the impact force of sand by combing Eq. (8) with Eqs. (2), (4), and (5).

$$
\tau^{\prime}=\left\{\frac{\left(\cot ^{2} \alpha-\cot ^{2} \varphi\right)\left(\cot \varphi+\frac{C_{\mathrm{L}}}{C_{\mathrm{D}}}\right) \sin \alpha}{\frac{C_{\mathrm{L}}}{C_{\mathrm{D}}} \cot \alpha+\cot \varphi \sqrt{\cot ^{2} \alpha-\cot ^{2} \varphi+\left(\frac{C_{\mathrm{L}}}{C_{\mathrm{D}}}\right)^{2}}}\right\} \cdot \tau .
$$

In this analysis, the impact force of sand on the slope is related to grain diameter, slope angle, angle of repose, the resistance of overland flow, etc. When $\theta$ is $90^{\circ}$ or $0^{\circ}$, the water-sand flow moves downward or laterally, respectively. When it varies between $0^{\circ}$ and $90^{\circ}$, the flow morphology is referred to as "tree root," which means that the flow moves along the edge of the sand debris flow. The accumulation area is presented as an inverted "goblet or fan." This phenomenon is consistent with field observations.

The saturated unit weight of sand $\left(\gamma_{\mathrm{s}}\right)$ is $2.5 \times 10^{4}$ $\mathrm{N} / \mathrm{m}^{3}$, and the unit weight of water $\left(\gamma_{\mathrm{w}}\right)$ is $1.0 \times 10^{4} \mathrm{~N} / \mathrm{m}^{3}$. The diameter of sand particles $(d)$ is assumed to be $1 \mathrm{~mm}$, and the internal friction angle $\varphi$ is $37^{\circ}$. Seepage pressure is not considered here. The value of $\theta$ is set to $90^{\circ}, f_{1}\left(\frac{U^{*} d}{v}\right)$ is assumed to be 0.05 , and the slope angle of the sand slope $(\alpha)$ is set to $34^{\circ}$. By cooperative solving Eqs. (8) and (13), we obtained the drag force required to initiate a water-sand flow to be $0.1 \mathrm{~N} / \mathrm{cm}^{2}$.

\section{Sand debris flow model}

Modeling of sand slopes has previously been performed by the Institute of Mountain Hazards and Environment [2, 12-14], CAS, the Chengdu University of Technology

$$
\begin{aligned}
& \tau^{\prime}=\left\{\left(1+\frac{C_{\mathrm{L}}}{C_{\mathrm{D}}} \tan \varphi\right)\left(\cos ^{2} \alpha-\sin ^{2} \alpha \tan ^{2} \varphi\right)\right\} \cdot\left\{\left(\frac{F_{\mathrm{L}}}{F_{\mathrm{D}}} \cos \alpha-\sin \alpha \sin \theta \cot ^{2} \varphi\right)\right. \\
& \left.+\cot \varphi \sqrt{\cos ^{2} \alpha-\sin ^{2} \alpha \tan ^{2} \varphi+\left(\frac{F_{\mathrm{L}}}{F_{\mathrm{D}}}\right)^{2} \cos ^{2} \alpha+2 \frac{F_{\mathrm{L}}}{F_{\mathrm{D}}} \sin \alpha \cos \alpha \sin \theta+\sin ^{2} \alpha \sin ^{2} \theta \cot ^{2} \varphi}\right\}^{-1} \cdot \tau \text {. }
\end{aligned}
$$

When $\theta=90^{\circ}$, the direction of water flow is the same as the downward orientation of the slope surface. Equation (13) is then obtained from the simplified Eq. (12).

$\tau^{\prime}=\left(\cos \alpha-\frac{\sin \alpha}{\tan \varphi}\right) \tau$.

When $\theta=0^{\circ}$, the direction of water flow is orthogonal to the slope surface and the water-sand flow moves laterally. Equation (14) is then obtained from the simplified Eq. (12).
[15], Southwest Jiaotong University [16], and other institutions, focused on studies of stability and flow features of dry sand on sand slopes. However, all of them were based on ideal models under laboratory conditions, and none involved an analysis of precipitation. In this study, we built a sand debris flow model in a scale of 1:100 to further evaluate flow formation mechanisms and characteristics and verified the theoretical analysis with field observations. An undisturbed sand sample from the sand debris flow was used to build the analog model using field data and Google earth satellite images of the sand debris flow. A schematic 


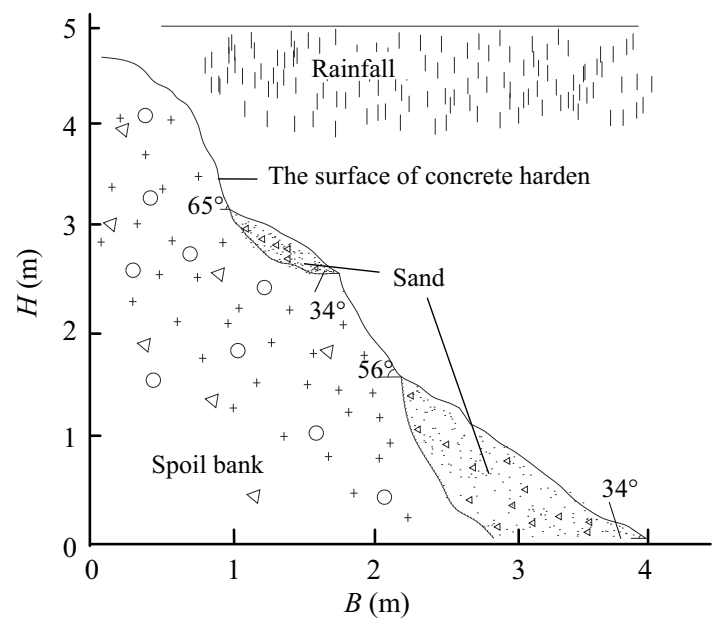

(a)

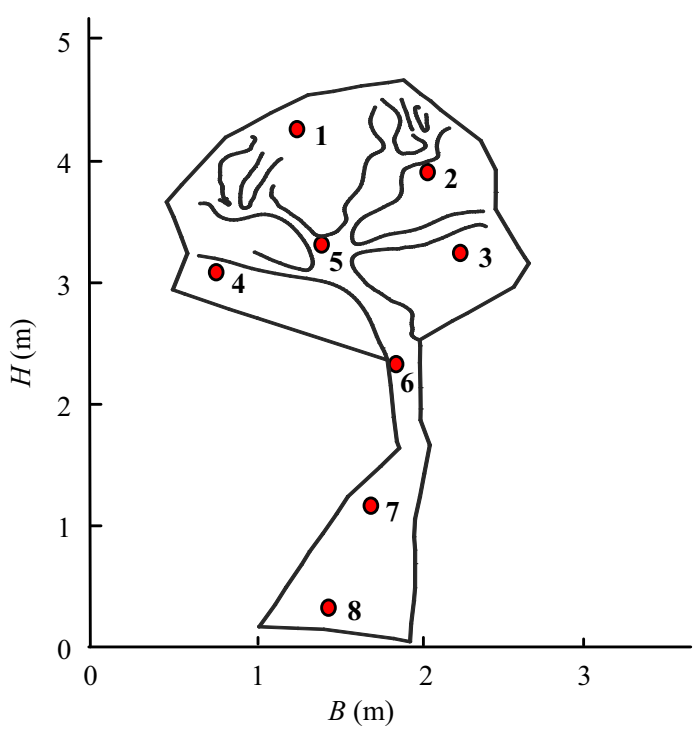

(b)

Fig. 3 Schematic diagrams of the model (a) and locations of rainfall collection (b)

diagram of the model is shown in Fig. 3a. Different grainsize compositions and precipitation amounts were analyzed to determine the threshold value for water-sand flow initiation, the flow characteristics of water-sand flow, and the relationship between the rainfall amount and duration and the sand grain size.

\subsection{Experimental preparation}

Slope length, width, angle, and other parameters were calculated using field data and satellite images of the study area. The model comprised large waste banks to ensure the geometric and environmental similarity between the experimental slope and the sand debris flow. Based on the size of the waste banks, the sand debris flow model was established in a scale of 1:100. The source region and accumulation area of the sand debris flow model are shown in Fig. 4. The source region of the sand debris flow was $2.78 \mathrm{~m}$ long and $2.33 \mathrm{~m}$ wide, with a slope of $39^{\circ}-78^{\circ}$ and circumference of $10.17 \mathrm{~m}$. The sand-sliding region was $0.58 \mathrm{~m}$ long and $0.11 \mathrm{~m}$ wide, with a slope angle of $43^{\circ}-$ $58^{\circ}$. The slopes of the steep surfaces on both sides of the sand-sliding region were $50^{\circ}-75^{\circ}$. The accumulation area was $1.56 \mathrm{~m}$ long and $0.23-1.01 \mathrm{~m}$ wide, with a slope angle of $33^{\circ}-35^{\circ}$. To ensure that the formation and sand-sliding regions were similar to that of the field bedrock, cement mortar was used to harden the material and to make the surface rough. The overall shape and environmental conditions of the model were almost equivalent to those of Ranwu-Songzong section. For model analysis, $1.2 \mathrm{~m}^{3}$ of weathered sand from the original granite sand debris flow was used.

The formation mechanisms and flow characteristics of water-sand flows are closely related to rainfall duration, rainfall intensity, and sand particle size. Thus, rainfall conditions and their observation and recording methods are also very important. In this study, the rainfall system was a purpose-built artificial stimulation rainfall device. Rainfall intensity was divided into heavy rainfall $(0.8 \mathrm{~mm} / \mathrm{min})$, moderate rainfall $(0.5 \mathrm{~mm} / \mathrm{min})$, and light rainfall $(0.3 \mathrm{~mm} / \mathrm{min})$ [20], and the rainfall was controlled by pressure. Five rain collectors were placed in the source region of the model, one collector was placed in the sandsliding region, and two collectors were placed in the accumulation area (Fig. 3b). To guarantee uniform operation of the rainfall device and ensure that the threshold value of water-sand flow initiation was not influenced, the eight rain collectors were controlled within the limits of experimental errors. Dual HD close-up observation cameras were operated simultaneously in the source region, sand-sliding region, and accumulation area.

\subsection{Experimental process}

Before the experiment, dry sand was added to the model according to field data. The sand was painted red and placed in the water-sand flow initiation region. Thus, we guaranteed that the geometry and environmental characteristics of sand in the source region, sand-sliding region, and accumulation area were consistent with the field. Then, rain collectors and cameras were placed at different positions on the slope. After preparation, the cameras were switched on, and the rainfall device was operated until initiation of water-sand flow. The hysteresis time until the water-sand flow ceased was simultaneously recorded, and the rain collectors were removed. The experiment was repeated three times for each rainfall intensity. Wind-free and sunny weather was required to ensure accuracy of the 

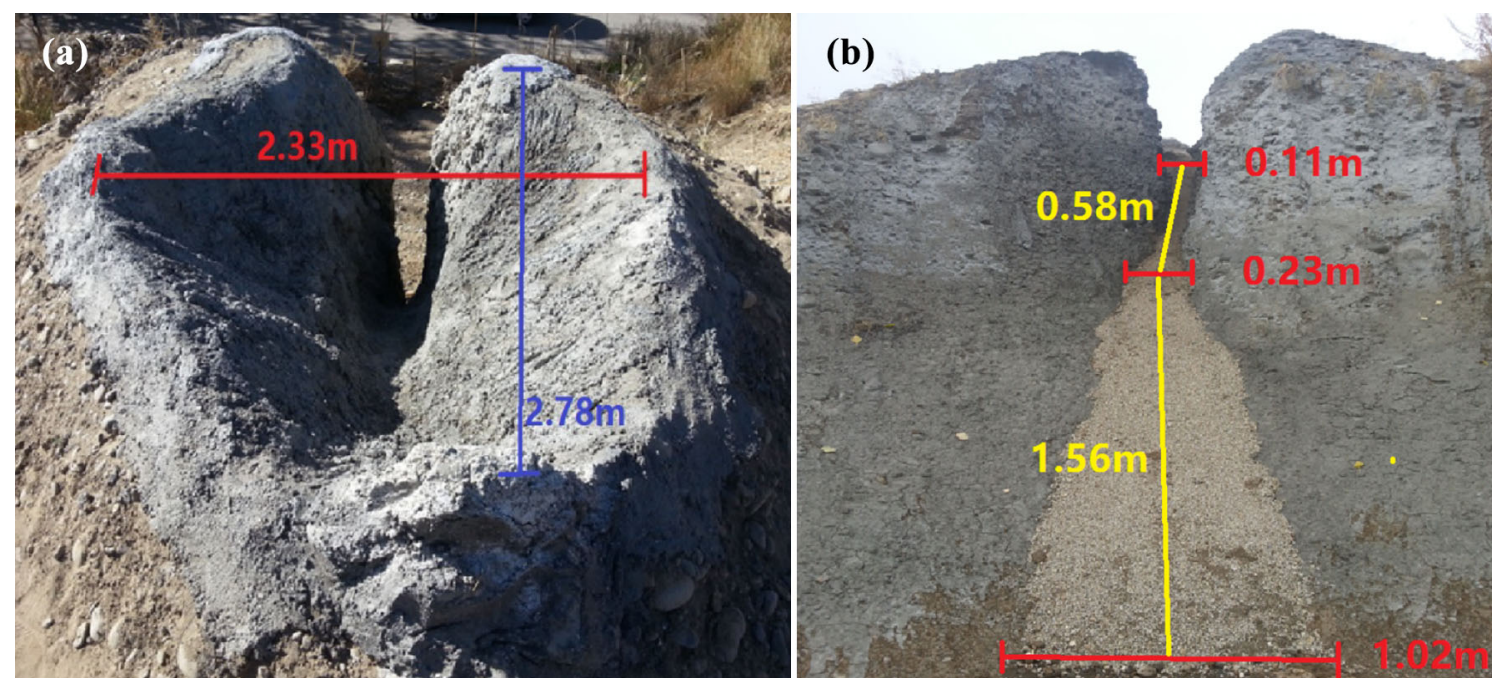

Fig. 4 Source region (a) and accumulation area of the sand debris flow model (b)

Table 2 Rainfall amount threshold for water-sand flow initiation (mm)

\begin{tabular}{|c|c|c|c|c|c|c|c|c|c|}
\hline \multirow[t]{2}{*}{ Rain collection device no. } & \multicolumn{3}{|c|}{ Light rainfall } & \multicolumn{3}{|c|}{ Moderate rainfall } & \multicolumn{3}{|c|}{ Heavy rainfall } \\
\hline & Group 1 & Group 2 & Group 3 & Group 1 & Group 2 & Group 3 & Group 1 & Group 2 & Group 3 \\
\hline 1 & 11.2 & 10.9 & 11.2 & 7.8 & 7.8 & 7.5 & 4.1 & 4.1 & 4.1 \\
\hline 2 & 10.8 & 11.1 & 10.9 & 7.6 & 7.7 & 7.7 & 4.2 & 4.1 & 4.2 \\
\hline 3 & 11.0 & 11.3 & 11.2 & 7.8 & 7.5 & 7.5 & 4.1 & 4.2 & 4.1 \\
\hline 4 & 11.1 & 11.2 & 10.8 & 7.5 & 7.6 & 7.6 & 4.1 & 4.1 & 4.2 \\
\hline 5 & 10.9 & 10.8 & 10.9 & 7.7 & 7.7 & 7.7 & 4.2 & 4.2 & 4.1 \\
\hline 6 & 10.8 & 11.0 & 11.2 & 7.7 & 7.5 & 7.5 & 4.1 & 4.1 & 4.1 \\
\hline 7 & 11.3 & 11.3 & 11.3 & 7.5 & 7.5 & 7.6 & 4.1 & 4.2 & 4.2 \\
\hline 8 & 11.2 & 10.9 & 10.9 & 7.7 & 7.7 & 7.5 & 4.2 & 4.1 & 4.1 \\
\hline
\end{tabular}

Table 3 Rainfall duration threshold for water-sand flow initiation

\begin{tabular}{llll}
\hline Rainfall intensity & Group no. & Rainfall duration (min) & Hysteresis time (s) \\
\hline Light rainfall & Group 1 & 36.7 & 53 \\
& Group 2 & 36.9 & 53 \\
Moderate rainfall & Group 3 & 36.8 & 54 \\
& Group 1 & 15.7 & 37 \\
Heavy rainfall & Group 2 & 15.8 & 36 \\
& Group 3 & 15.9 & 38 \\
& Group 1 & 5.2 & 16 \\
& Group 2 & 5.2 & 17 \\
\hline
\end{tabular}

experiment. Before analyzing the relationship between grain-size composition and rainfall duration and intensity, the different sand grain sizes were first determined. Particle diameters were divided into $\leq 0.25 \mathrm{~mm}, 0.25-2 \mathrm{~mm}$, and $\geq 2 \mathrm{~mm}$. Then, sand particles were mixed according to different grain compositions (Table 4).

\subsection{Experimental results and analysis}

The rainfall amounts and durations required for water-sand flow initiation on the analog sand debris flow under different precipitation conditions are given in Tables 2 and 3. The average rainfall amounts required to initiate water- 

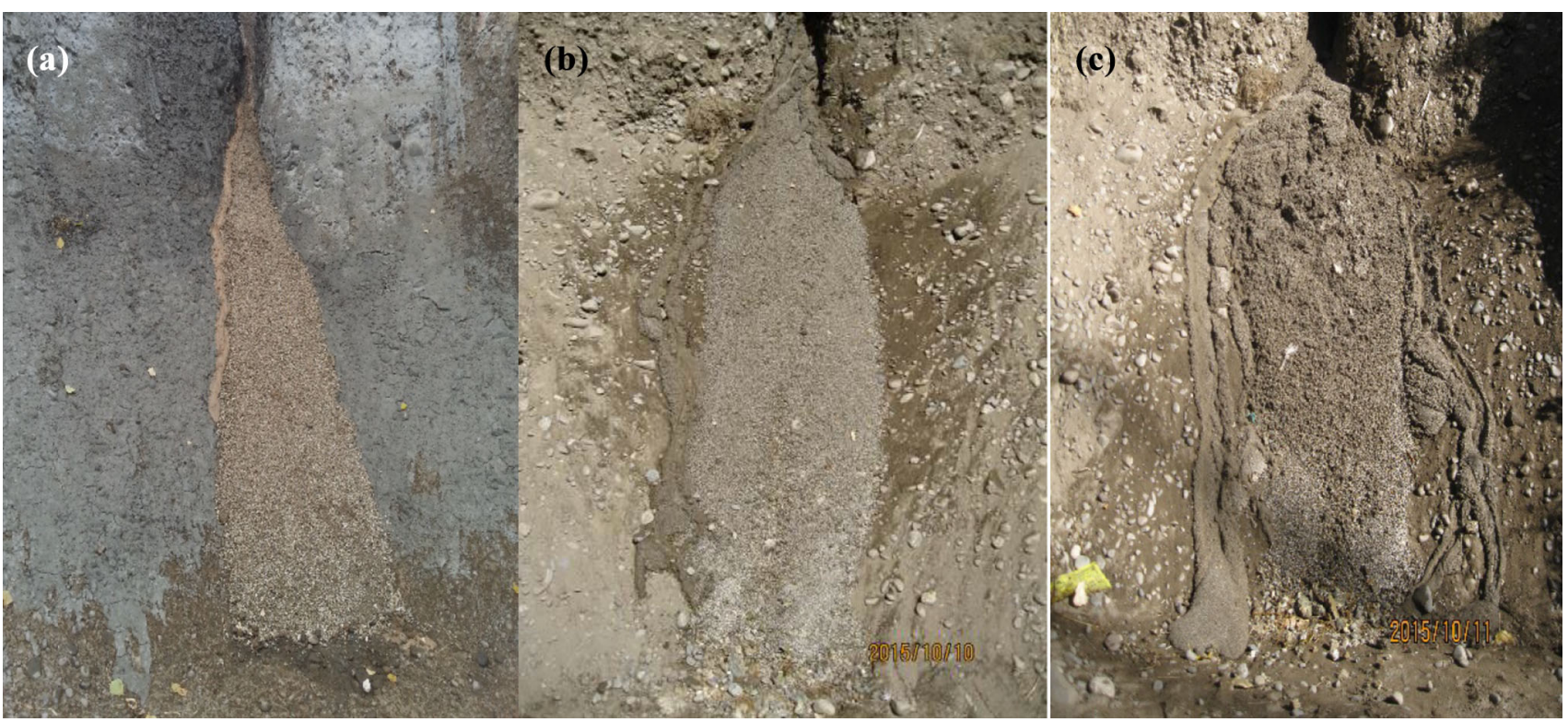

Fig. 5 Images of water-sand flow initiation under different rainfall intensity: light rainfall (a), moderate rainfall (b), and heavy rainfall (c)

Table 4 Effect of rainfall amount and duration thresholds on the grain-size composition of water-sand flows

\begin{tabular}{llllll}
\hline Sample no. & \multicolumn{2}{l}{ Sand composition and ratio $(\%)$} & Rainfall (mm) & Rainfall time (min) \\
\cline { 2 - 5 } & $<0.25 \mathrm{~mm}$ & $0.25-2 \mathrm{~mm}$ & $>2 \mathrm{~mm}$ & 8.6 & 17.2 \\
1 & 10 & 70 & 20 & 7.3 & 14.6 \\
2 & 20 & 65 & 15 & 6.0 & 12.0 \\
3 & 30 & 60 & 5 & 5.2 & 9.4 \\
5 & 40 & 55 & 3 & 4.5 & 7.1 \\
6 & 50 & 47 & 1 & 3.7 & 7.5 \\
\hline
\end{tabular}

Table 5 Comparison in conclusions of sample test, theoretical analysis, and model test

\begin{tabular}{ll}
\hline Method & Conclusion \\
\hline $\begin{array}{c}\text { Theoretical } \\
\text { analysis }\end{array}$ & Sand stability was influenced by moisture content and the external force \\
$\begin{array}{c}\text { Sample test } \\
\text { Model test }\end{array}$ & $\begin{array}{c}\text { At rainfall amounts less than that required to initiate water-sand flow, sand was more stable. However, the force of raindrops } \\
\text { also caused the sand to lose stability }\end{array}$ \\
\hline
\end{tabular}

sand flow were $11.05,7.88$, and $4.14 \mathrm{~mm}$ for light rainfall, moderate rainfall, and heavy rainfall, respectively. The average rainfall durations required to initiate water-sand flow were $36.8,15.8$, and $5.2 \mathrm{~min}$, respectively, and the average hysteresis time was 53.3, 37.0, and $16.7 \mathrm{~s}$, respectively.

Thus, the maximum rainfall amount and duration required for water-sand flow initiation, as well as the longest hysteresis time, were obtained under light rainfall conditions. Water-sand flow was initiated by the lowest rainfall amount and duration under heavy rainfall conditions, and the hysteresis time was the shortest. Moreover, the water-sand flow increased with the rainfall intensity increasing (Fig. 5), probably because of the reduced surface water infiltration and evaporation. Combined with field observation data, it is clear that water-sand flows form easily in short duration under heavy rainfall. Therefore, $4.14 \mathrm{~mm}$ (the threshold rainfall under heavy rainfall) was 
chosen as the threshold value for the initiation of a watersand flow.

Table 4 shows that an increase in the percentage of sand particles $\leq 0.25 \mathrm{~mm}$ leads to a decrease in the rainfall amount and duration required to induce water-sand flow. The same is true for the decrease in the abundance of sand particles $\geq 0.25 \mathrm{~mm}$. For the same rainfall intensity, the threshold values for water-sand flow initiation decrease with rainfall duration decreasing. According to experimental observations, the permeability of the sand decreases with an increase in fine particle content. Surface runoff then forms in a shorter rainfall duration. Moreover, smaller sand is extremely easily washed away. Therefore, the finer the particles, the less rainfall required to initiate watersand flow. When the percentage of fine sand stays the same, a small water-sand flow forms first on the steepest slope.

\section{Comparison and discussion}

Sand was more stable under moist conditions than dry conditions. With no external forces and dry conditions, the sand was at its stability limit. When subjected to external forces such as vibrations, the sand lost stability and began sliding. A comparison of samples 1, 2, 4, and 5 in Table 1 indicates that the moisture content, cohesion, and internal friction angle increased under light rainfall. Sand stability in the modeling experiment was thus consistent with that of the theoretical analysis and field data. It was also influenced by the force of raindrops. A full comparison is given in Table 5.

Theoretical analysis suggested that the water-sand flow moved downward when $\theta$ was $90^{\circ}$ and laterally when $\theta$ was $0^{\circ}$. When the value of $\theta$ varied between $0^{\circ}$ and $90^{\circ}$, the water-sand flow moved downward along the locally steeper region of the slope. A sand head formed rapidly at the front of the debris flow. When the resistance of the sand head exceeded the impact force of the water-sand flow, the water-sand flow changed to move laterally. With the rapid formation of a smaller sand head on the lateral portions of the slope, the water-sand flow was once again forced to move downward. This cycle resulted in a "tree root" morphology of the water-sand flow, whereby it moved along the edge of the accumulation area of the sand debris flow. Thus, the accumulation area had an inverted "goblet or fan" shape. These results are consistent with both the theoretical analysis and the field observations.

\section{Conclusions}

In this study, we performed the laboratory analog modeling of a sand debris flow under different precipitation conditions, according to the theory of equilibrium forces and hydrological dynamics. The following conclusions were obtained:

1. According to the analog model, the effect of permeability decreased with an increase in rainfall intensity. The rainfall duration and amount required for formation of a water-sand flow also decreased. Therefore, water-sand flows are more easily formed in short duration under heavy rainfall.

2. For the same rainfall intensity, the rainfall amount and duration required for formation of a water-sand flow decreased with an increase in the abundance of sand particles smaller than $0.25 \mathrm{~mm}$. Therefore, water-sand flows are more easily initiated with a greater fine sand particle content.

3. During the process of downward water-sand flow, a sand head rapidly formed at the front of the flow. The water-sand flow was thus forced to alternate between lateral and downward flow, resulting in a "tree root" pattern as it moved along the edge of the accumulation area of the sand debris flow. Our modeling results are consistent with field observations and theoretical analysis.

Acknowledgements This research was supported by the National Natural Science Foundation of China (NSFC) (Grants Nos. 41662020 and 41462012).

Open Access This article is distributed under the terms of the Creative Commons Attribution 4.0 International License (http:// creativecommons.org/licenses/by/4.0/), which permits unrestricted use, distribution, and reproduction in any medium, provided you give appropriate credit to the original author(s) and the source, provide a link to the Creative Commons license, and indicate if changes were made.

\section{References}

1. Zhu PY, He ZW, Wang YC (1999) A study of typical mountain hazards along Sichuan-Tibet Highway. Science and Technology University Press, Chengdu (in Chinese)

2. Zhang XG, Yang TJ, Tian JC (2013) The comprehensive prevention and control measures of debris flow along the SichuanTibet Highway. Geol Bull China 32(12):2031-2037 (in Chinese)

3. Sharpe CFS (1938) Landslides and related phenomena. Columbia University Press, New York

4. Caine N (1980) The rainfall intensity-duration control of shallow landslides and debris flows. Geogr Ann 62(1/2):23-27

5. Brunsden D, Chandler JH (1996) Development of an episodic landform change model based upon the Black Ven Mudslide, 
1946-1995. In: British Geomorphological Research Group Symposia Series 12, pp 869-896

6. Gabriels D (1999) The effect of slope length on the amount and size distribution of eroded silt loam soils: short slope laboratory experiments on interrill erosion. Geomorphology 28(28):169-172

7. Yoo C (2001) Laboratory investigation of bearing capacity behavior of strip footing on geogrid-reinforced sand slope. Geotext Geomembr 19(5):279-298

8. Pietronero L, Tartaglia P, Zhang YC (2006) Theoretical studies of self-organized criticality. Phys Stat Mech Appl 369(1):29-70

9. Mohanty PK, Dhar D (2007) Critical behavior of sand pile models with sticky grains. Phys Stat Mech Appl 384(1):34-38

10. Lou DF, Mao JZ, Zhu PY (1995) Mountain disasters and prevention countermeasures in Sichuan-Tibet highway south (Tibet). Science Press, Beijing (in Chinese)

11. Wu GX, Zeng RB, Wang $\mathrm{CH}$ et al (2006) The inducing factors and the collapse conditions of sand debris flow. China Railw Sci 27(05):7-12 (in Chinese)

12. Wang $\mathrm{CH}$, Zhang $\mathrm{XG}$, Que $\mathrm{Y}$ et al (2007) Formation and basic characteristics of sand debris flow composed of granular clasts: part I of sand debris flows eries. Rock Soil Mech 28(01):29-35 (in Chinese)
13. Wang CH, Que Y, Li XP et al (2007) Movement characteristics and dynamical numerical analysis of sand debris flow composed by granular clastics. Rock Soil Mech 28(2):219-223 (in Chinese)

14. Wang CH, Que Y, Xu J et al (2007) Equation of motion and soil pressure for sand debris flow composed by granular clast. Rock Soil Mech 28(7):1300-1303 (in Chinese)

15. Zhang YC, Huang RQ, Fu RH et al (2010) Experimental research on dynamic failure mechanism of large-scale talus slope. Chin J Rock Mech Eng 29(01):65-72 (in Chinese)

16. Jiang LW, Yao LK, Jiang ZX et al (2004) Experiments on Sand debris flow and the Countermeasure. J Mt Sci 22(1):97-103 (in Chinese)

17. Que Y, Wang CH, Zhang XG (2003) Formative mechanism and control of typical sand debris flow along Sichuan-Tibet Highway. J Mt Sci 21(05):595-598 (in Chinese)

18. Deangeli C (2008) Laboratory Granular Flows generated by Slope Failures. Rock Mech Rock Eng 41(1):199-217

19. Abdulkhanova DR, Grigor'ev VY (2009) Generalized equations of sediment discharge for assessing the erosion and transport capacities of shallow surface flows. Moscow Univ Soil Sci Bull 64(3):138-144

20. Cui B, Su F, Guo C (2012) Erosion experiment of construction spoil with different particle gradation under simulated rainfall condition. Sci Soil Water Conser 10(02):61-65 (in Chinese) 